\title{
Norm inequalities of operators and commutators on generalized weighted morrey spaces
}

\author{
Yue $\mathrm{Hu}^{\mathrm{a}, *}$, Yueshan Wang ${ }^{\mathrm{b}}$ \\ ${ }^{a}$ School of Mathematics and Information, Henan Polytechnic University, Jiaozuo 454003, P. R. China. \\ ${ }^{b}$ Department of Mathematics, Jiaozuo University, Jiaozuo 454003, P. R. China.
}

Communicated by X.-J. Yang

\begin{abstract}
We prove that, if a class of operators, which includes singular integral operator with rough kernel, Bochner-Riesz operator and Marcinkiewicz integral operator, are bounded on weighted Lebesgue spaces and satisfy some local pointwise control, then these operators and associated commutators, formed by a BMO function and these operators, are also bounded on generalized weighted Morrey spaces. (C)2017 All rights reserved.
\end{abstract}

Keywords: Singular integral with rough kernel, Bochner-Riesz operator, Marcinkiewicz integral, commutator, weighted Morrey space.

2010 MSC: 42B35, 47B38, 30H35.

\section{Introduction and results}

The classical Morrey space was introduced by Morrey [10] in 1938. It plays an important role in the theory of partial differential equations. Morrey space is defined by

$$
\mathrm{L}^{\mathrm{p}, \lambda}\left(\mathbb{R}^{\mathrm{n}}\right)=\left\{f \in \mathrm{L}_{\text {loc }}^{\mathrm{p}}\left(\mathbb{R}^{\mathrm{n}}\right):\|f\|_{\mathrm{L}^{p, \lambda}}<\infty\right\},
$$

where

$$
\|\mathbf{f}\|_{L^{p, \lambda}\left(\mathbb{R}^{n}\right)}=\sup _{x \in \mathbb{R}^{n}, r>0} r^{-\lambda / p}\|f\|_{L^{p}(B(x, r))}<\infty .
$$

Note that $L^{p, 0}\left(\mathbb{R}^{\mathfrak{n}}\right)=\mathrm{L}^{\mathrm{p}}\left(\mathbb{R}^{\mathfrak{n}}\right)$ and $\mathrm{L}^{\mathrm{p}, \mathrm{n}}\left(\mathbb{R}^{\mathfrak{n}}\right)=\mathrm{L}^{\infty}\left(\mathbb{R}^{\mathfrak{n}}\right)$. If $\lambda<0$ or $\lambda>\mathfrak{n}$, then $\mathrm{L}^{\mathrm{p}, \lambda}\left(\mathbb{R}^{\mathfrak{n}}\right)=\Theta$, where $\Theta$ is the set of all functions equivalent to 0 on $\mathbb{R}^{n}$.

Let $\Phi(r), r>0$ be a growth function, that is, a positive increasing function in $(0, \infty)$, which satisfies doubling condition

$$
\Phi(2 r) \leqslant D \Phi(r), \quad \forall r>0
$$

\footnotetext{
*Corresponding author

Email addresses: huu3y6@163.com (Yue Hu), wangys1962@163.com (Yueshan Wang)
}

doi:10.22436/jnsa.010.07.12 
where $\mathrm{D}=\mathrm{D}(\Phi) \geqslant 1$ is a doubling constant independent of $r$. In [9] Mizuhara gave a generalization Morrey space $L^{p, \Phi}\left(\mathbb{R}^{n}\right)$ considering $\Phi(r)$ instead of $r^{\lambda}$ in (1.1).

Komori and Shirai [8] introduced a version of the weighted Morrey space $L^{p, k}\left(\omega, \mathbb{R}^{n}\right)$, which is a natural generalization of the weighted Lebesgue space $L^{p}\left(\omega, \mathbb{R}^{n}\right)$.

Let $1 \leqslant p<\infty, 0<\kappa<1$ and $\omega$ be a weight function. Then the space $L^{p, k}\left(\omega, \mathbb{R}^{\mathfrak{n}}\right)$ is defined by

$$
\mathrm{L}^{\mathrm{p}, \mathrm{k}}\left(\omega, \mathbb{R}^{\mathrm{n}}\right)=\left\{\mathrm{f} \in \mathrm{L}_{\mathrm{loc}}^{\mathrm{p}}(\omega):\|f\|_{\mathrm{L}^{\mathrm{p}, \mathrm{k}}\left(\omega, \mathbb{R}^{\mathrm{n}}\right)}<\infty\right\},
$$

where

$$
\|f\|_{L^{p, k}\left(\omega, \mathbb{R}^{n}\right)}=\sup _{x \in \mathbb{R}^{n}, r>0}\left(\frac{1}{\omega(B(x, r))^{\kappa}} \int_{B(x, r)}|f(y)|^{p} d y\right)^{\frac{1}{p}} .
$$

Let $1 \leqslant p<\infty, \varphi$ be a positive measurable function on $\mathbb{R}^{n} \times(0, \infty)$ and $\omega$ be a non-negative measurable function on $\mathbb{R}^{n}$. We denote by $M_{\varphi}^{p}\left(\omega, \mathbb{R}^{n}\right)$ the generalized weighted Morrey space, the space of all functions $f \in L_{\text {loc }}^{p}(\omega)$ with finite norm

$$
\|f\|_{M_{\varphi}^{p}\left(w, \mathbb{R}^{\mathfrak{n}}\right)}=\sup _{\chi \in \mathbb{R}^{n}, r>0} \frac{1}{\varphi(x, r)}\left(\frac{1}{w(B(x, r))}\|f\|_{L^{p}(\omega, B(x, r))}^{p}\right)^{1 / p},
$$

where

$$
\|f\|_{L^{p}(w, B(x, r))}=\left(\int_{B(x, r)}|f(y)|^{p} \mathcal{W}(y) d y\right)^{1 / p} .
$$

If $\omega=1$ and $\varphi(x, r)=r^{\frac{\lambda-n}{p}}$ with $0 \leqslant \lambda \leqslant n$, then $M_{\varphi}^{p}\left(\omega, \mathbb{R}^{n}\right)=L^{p, \lambda}\left(\mathbb{R}^{n}\right)$ is the classical Morrey space. If $\varphi(x, r)=\omega(B(x, r))^{\frac{k-1}{p}}$, then $M_{\varphi}^{p}\left(\omega, \mathbb{R}^{\mathfrak{n}}\right)=L^{p, \kappa}\left(\omega, \mathbb{R}^{\mathfrak{n}}\right)$ is the weighted Morrey space.

In this paper, we prove that, if a class of operators are bounded on weighted Lebesgue space and satisfy some local pointwise control, then these operators and associated commutators, formed by a BMO function and these operators, are also bounded on generalized weighted Morrey space. Our main results can be formulated as follows.

Theorem 1.1. Let $1 \leqslant s^{\prime} \leqslant p<\infty, \omega \in A_{p / s^{\prime}}$ and $T$ be a sublinear operator which satisfies

$$
\sup _{x \in B\left(x_{0}, l\right)}\left|T\left(f \chi_{\left(B\left(x_{0}, 2 l\right) c\right.}\right)(x)\right| \leqslant C \sum_{j=1}^{\infty}\left(\frac{1}{\left|B\left(x_{0}, 2^{j+1} l\right)\right|} \int_{B\left(x_{0}, 2^{j+1} l\right)}|f(z)|^{s^{\prime}} d z\right)^{1 / s^{\prime}},
$$

for any $x_{0} \in \mathbb{R}^{n}$ and $l>0$.

(i) Suppose $\left(\varphi_{1}, \varphi_{2}\right)$ satisfies the condition

$$
\int_{l}^{\infty} \frac{\underset{r<t<\infty}{\operatorname{essinf}} \varphi_{1}\left(x_{0}, t\right) \omega\left(B\left(x_{0}, t\right)\right)^{\frac{1}{p}}}{\omega\left(B\left(x_{0}, r\right)\right)^{\frac{1}{p}}} \frac{d r}{r} \leqslant c_{0} \varphi_{2}\left(x_{0}, l\right),
$$

where $\mathrm{c}_{0}$ does not depend on $\mathrm{x}$ and $\mathrm{r}$. If $\mathrm{T}$ is bounded on $\mathrm{L}^{\mathrm{p}}\left(\omega, \mathbb{R}^{\mathrm{n}}\right)$ for $\mathrm{p}>1$, then $\mathrm{T}$ is also bounded from $M_{\varphi_{1}}^{p}\left(\omega, \mathbb{R}^{n}\right)$ to $M_{\varphi_{2}}^{p}\left(\omega, \mathbb{R}^{n}\right)$ and

$$
\|T f\|_{M_{\varphi_{2}}^{p}\left(\omega, \mathbb{R}^{n}\right)} \leqslant C\|f\|_{M_{\varphi_{1}}^{p}\left(\omega, \mathbb{R}^{n}\right)} .
$$

(ii) Suppose $\left(\varphi_{1}, \varphi_{2}\right)$ satisfies the condition

$$
\int_{l}^{\infty}\left(1+\ln \frac{r}{l}\right) \frac{\underset{r}{\operatorname{essinf}<t<\infty} \varphi_{1}\left(x_{0}, t\right) \omega\left(B\left(x_{0}, t\right)\right)^{\frac{1}{p}}}{\omega\left(B\left(x_{0}, r\right)\right)^{\frac{1}{p}}} \frac{d r}{r} \leqslant c_{0} \varphi_{2}\left(x_{0}, l\right),
$$


where $\mathrm{c}_{0}$ does not depend on $\mathrm{x}_{0}$ and l. If $\mathrm{b} \in \mathrm{BMO}\left(\mathbb{R}^{\mathrm{n}}\right)$, and $[\mathrm{b}, \mathrm{T}]$ is bounded on $\mathrm{L}^{\mathrm{p}}\left(\omega, \mathbb{R}^{\mathrm{n}}\right)$ for $1<\mathrm{p}<\infty$, then $[\mathrm{b}, \mathrm{T}]$ is also bounded from $\mathrm{M}_{\varphi_{1}}^{\mathrm{p}}\left(\omega, \mathbb{R}^{\mathrm{n}}\right)$ to $\mathrm{M}_{\varphi_{2}}^{\mathrm{p}}\left(\omega, \mathbb{R}^{\mathrm{n}}\right)$ and

$$
\|[b, T] f\|_{M_{\varphi_{2}}^{p}\left(\omega, \mathbb{R}^{n}\right)} \leqslant C\|b\|_{*}\|f\|_{M_{\varphi_{1}}^{p}\left(\omega, \mathbb{R}^{n}\right)} .
$$

Remark 1.2. Let $\varphi_{1}(x, t)=\left(\Phi(t) t^{-n}\right)^{\frac{1}{p}}$, let $\varphi_{2}(x, t)=(\Phi(t))^{\frac{1}{p}} t^{\frac{n}{q}}$, and let $\omega=1$. If $1 \leqslant D(\Phi) \leqslant 2^{n}$, it is easy to prove $\left(\varphi_{1}, \varphi_{2}\right)$ satisfies the conditions (1.3) and (1.4).

Remark 1.3. Let $\varphi_{1}\left(x_{0}, t\right)=\varphi_{2}\left(x_{0}, t\right)=\omega\left(B\left(x_{0}, t\right)\right)^{\frac{k-1}{p}}, 0<\kappa<1$, and $w \in A_{\infty}\left(\mathbb{R}^{\mathfrak{n}}\right)$, then $\left(\varphi_{1}, \varphi_{2}\right)$ satisfies the conditions (1.3) and (1.4).

Then we have the following corollaries.

Corollary 1.4. Let $1 \leqslant s^{\prime} \leqslant p<\infty, \omega \in A_{p / s^{\prime}}, 1 \leqslant D(\Phi) \leqslant 2^{n}$, and let $T$ be a sublinear operator which satisfies (1.4) for any $x_{0} \in \mathbb{R}^{n}$ and $l>0$. If $\mathrm{T}$ is bounded on $\mathrm{L}^{\mathrm{p}}\left(\omega, \mathbb{R}^{\mathrm{n}}\right)$ for $\mathrm{p}>1$, then $\mathrm{T}$ is bounded on $\mathrm{L}^{\mathrm{p}, \Phi}\left(\mathbb{R}^{\mathrm{n}}\right)$. If $\mathrm{b} \in \mathrm{BMO}\left(\mathbb{R}^{\mathfrak{n}}\right)$, and $[\mathrm{b}, \mathrm{T}]$ is bounded on $\mathrm{L}^{\mathrm{p}}\left(\omega, \mathbb{R}^{\mathfrak{n}}\right)$ for $\mathrm{p}>1$, then $[\mathrm{b}, \mathrm{T}]$ is also bounded on $\mathrm{L}^{\mathrm{p}, \Phi}\left(\mathbb{R}^{\mathfrak{n}}\right)$.

Corollary 1.5. Let $1 \leqslant s^{\prime} \leqslant p<\infty, \omega \in A_{p / s^{\prime}}, 0<\alpha<n$, and let $T$ be a sublinear operator which satisfies (1.4) for any $x_{0} \in \mathbb{R}^{n}$ and $l>0$. If $\mathrm{T}$ is bounded on $\mathrm{L}^{\mathrm{p}}\left(\omega, \mathbb{R}^{\mathfrak{n}}\right)$ for $p>1$, then $\mathrm{T}$ is bounded on $\mathrm{L}^{\mathrm{p}, \mathrm{k}}\left(\omega, \mathbb{R}^{\mathrm{n}}\right)$. If $\mathrm{b} \in \mathrm{BMO}\left(\mathbb{R}^{\mathrm{n}}\right)$, and $[\mathrm{b}, \mathrm{T}]$ is bounded on $\mathrm{L}^{\mathrm{p}}\left(\omega, \mathbb{R}^{\mathrm{n}}\right)$ for $\mathrm{p}>1$, then $[\mathrm{b}, \mathrm{T}]$ is also bounded on $\mathrm{L}^{\mathrm{p}, \mathrm{k}}\left(\omega, \mathbb{R}^{\mathrm{n}}\right)$.

This paper is organized as follows. Section 2 is devoted to prove some preliminary results. In Section 3 , we prove our main result and in Section 4 we give some applications to our main theorem.

\section{Some preliminaries}

We begin with some properties of $A_{p}$ weights which play a great role in the proofs of our main results.

A weight $\omega$ is a nonnegative, locally integrable function on $\mathbb{R}^{n}$. Let $B=B\left(x_{0}, r_{B}\right)$ denote the ball with the center $x_{0}$ and radius $r_{B}$ and let $\lambda B=B\left(x_{0}, \lambda r_{B}\right)$. For a given weight function $\omega$ and a measurable set $E$, we also denote the Lebesgue measure of $E$ by $|E|$ and set weighted measure by $\omega(E)=\int_{E} \omega(x) d x$. For any given weight function $\omega$ on $\mathbb{R}^{n}, X \subseteq \mathbb{R}^{n}$ and $0<p<\infty$, denote by $L^{p}(\omega, X)$ the space of all functions f satisfying

$$
\|f\|_{L^{p}(\omega, X)}=\left(\int_{X}|f(x)|^{p} \omega(x) d x\right)^{1 / p}<\infty .
$$

A weight $\omega$ is said to belong to $A_{p}\left(\mathbb{R}^{\mathfrak{n}}\right)$ for $1<p<\infty$, if there exists a constant

$$
\left(\frac{1}{|B|} \int_{B} \omega(x) d x\right)\left(\frac{1}{|B|} \int_{B} \omega(x)^{1-p^{\prime}} d x\right)^{p-1} \leqslant C
$$

where $p^{\prime}$ is the dual of $p$ such that $1 / p+1 / p^{\prime}=1$. The class $A_{1}\left(\mathbb{R}^{n}\right)$ is defined by

$$
\frac{1}{|B|} \int_{B} w(y) d y \leqslant C \cdot \underset{x \in B}{\operatorname{essinf}} w(x), \quad \text { for every ball } B \subset \mathbb{R}^{n} .
$$

By (2.1), we have

$$
\left(\omega^{-\frac{p^{\prime}}{p}}(B)\right)^{\frac{1}{p^{\prime}}}=\left\|\omega^{-\frac{1}{p}}\right\|_{L^{p^{\prime}}(B)} \leqslant C|B|(\omega(B))^{-\frac{1}{p}},
$$

for $1<p<\infty$.

Suppose $\omega \in A_{p}\left(\mathbb{R}^{\mathfrak{n}}\right), 1<p<\infty$, by the definition of $A_{p}\left(\mathbb{R}^{\mathfrak{n}}\right)$, we know that $\omega^{1-p^{\prime}} \in A_{p^{\prime}}\left(\mathbb{R}^{\mathfrak{n}}\right)$.

The classical $A_{\mathfrak{p}}\left(\mathbb{R}^{n}\right)$ weight theory was first introduced by Muckenhoupt in the study of weighted $\mathrm{L}^{\mathrm{p}}$-boundedness of Hardy-Littlewood maximal function in [11]. 
Following [7], a locally integrable function $b$ is said to be in $\operatorname{BMO}\left(\mathbb{R}^{\mathfrak{n}}\right)$ if

$$
\sup _{B \subset \mathbb{R}^{n}} \frac{1}{|B|} \int_{B}\left|b(x)-b_{B}\right| d x=\|b\|_{*}<\infty,
$$

where

$$
b_{B}=\frac{1}{|B|} \int_{B} b(y) d y
$$

Lemma 2.1 ([6]). Suppose $\omega \in A_{\infty}\left(\mathbb{R}^{n}\right)$ and $b \in \operatorname{BMO}\left(\mathbb{R}^{n}\right)$. Then for any $1 \leqslant p<\infty$ and $r_{1}, r_{2}>0$, we have

$$
\left(\frac{1}{\omega\left(B\left(x_{0}, r_{1}\right)\right)} \int_{B\left(x_{0}, r_{1}\right)}\left|b(x)-b_{B\left(x_{0}, r_{2}\right)}\right|^{p} \omega(x) d x\right)^{1 / p} \leqslant C\left(1+\left|\ln \frac{r_{1}}{r_{2}}\right|\right)\|b\|_{*} .
$$

In order to prove Theorem 1.1, we need to prove the following lemmas.

Lemma 2.2. Suppose that $1 \leqslant s^{\prime} \leqslant p<\infty, p>1$, and $\omega \in A_{p / s^{\prime}}\left(\mathbb{R}^{n}\right)$. If $T$ is bounded on $\mathrm{L}^{p}\left(\omega, \mathbb{R}^{n}\right)$ and satisfies (1.2), then for any $l>0$, there is a constant $C$ independent of $f$ such that

$$
\|T(f)\|_{L^{p}\left(\omega, B\left(x_{0}, l\right)\right)} \leqslant C \omega\left(B\left(x_{0}, l\right)\right)^{\frac{1}{p}} \int_{2 l}^{\infty}\|f\|_{L^{p}\left(\omega, B\left(x_{0}, r\right)\right)} \omega\left(B\left(x_{0}, r\right)\right)^{-\frac{1}{p}} \frac{d r}{r} .
$$

Proof. We write $f$ as $f=f_{1}+f_{2}$, where $f_{1}(y)=f(y) \chi_{B}\left(x_{0}, 2 l\right)(y), \chi_{B}\left(x_{0}, 2 l\right)$ denotes the characteristic function of $B\left(x_{0}, 2 l\right)$. Then

$$
\|T(f)\|_{L^{\mathfrak{p}}\left(w, B\left(x_{0}, l\right)\right)} \leqslant\left\|T\left(f_{1}\right)\right\|_{L^{\mathfrak{p}}\left(w, B\left(x_{0}, l\right)\right)}+\left\|T\left(f_{2}\right)\right\|_{L^{\mathfrak{p}}\left(\omega, B\left(x_{0}, l\right)\right)} .
$$

Since $f_{1} \in L^{p}\left(\omega, \mathbb{R}^{n}\right)$, from the boundedness of $T$ on $L^{p}\left(\omega, \mathbb{R}^{n}\right)(p>1)$ it follows that

$$
\begin{aligned}
\left\|T\left(f_{1}\right)\right\|_{L^{p}\left(\omega, B\left(x_{0}, l\right)\right)} & \leqslant\left\|T\left(f_{1}\right)\right\|_{L^{p}\left(\omega, \mathbb{R}^{\mathfrak{n}}\right)} \\
& \leqslant C\left\|f_{1}\right\|_{L^{p}\left(\omega, \mathbb{R}^{n}\right)} \\
& =C\|f\|_{L^{p}\left(\omega, B\left(x_{0}, 2 l\right)\right) .}
\end{aligned}
$$

By Hölder's inequality,

$$
\left|B\left(x_{0}, l\right)\right| \leqslant C \omega\left(B\left(x_{0}, l\right)\right)^{\frac{1}{p}}\left\|\omega^{-\frac{1}{p}}\right\|_{L^{p^{\prime}}\left(B\left(x_{0}, l\right)\right)} .
$$

Then, for any $p>1$,

$$
\begin{aligned}
\|f\|_{L^{p}\left(\omega, B\left(x_{0}, 2 l\right)\right)} & \leqslant C\left|B\left(x_{0}, l\right)\right|\|f\|_{L^{p}\left(\omega, B\left(x_{0}, 2 l\right)\right)} \int_{2 l}^{\infty} \frac{d r}{r^{\mathfrak{n}+1}} \\
& \leqslant C\left|B\left(x_{0}, l\right)\right| \int_{2 l}^{\infty}\|f\|_{L^{p}\left(\omega, B\left(x_{0}, r\right)\right)} \frac{d r}{r^{\mathfrak{n}+1}} \\
& \leqslant C \omega\left(B\left(x_{0}, l\right)\right)^{\frac{1}{p}}\left\|\omega^{-\frac{1}{\mathfrak{p}}}\right\|_{L^{p^{\prime}}\left(B\left(x_{0}, l\right)\right)} \int_{2 l}^{\infty}\|f\|_{L^{p}\left(\omega, B\left(x_{0}, r\right)\right)} \frac{d r}{r^{n+1}} \\
& \leqslant C \omega\left(B\left(x_{0}, l\right)\right)^{\frac{1}{p}} \int_{2 l}^{\infty}\|f\|_{L^{p}\left(\omega, B\left(x_{0}, r\right)\right.}\left\|\omega^{-\frac{1}{\mathfrak{p}}}\right\|_{L^{p^{\prime}}\left(B\left(x_{0}, r\right)\right)} \frac{d r}{r^{\mathfrak{n}+1}}
\end{aligned}
$$

Then, by (2.2) we get

$$
\left\|T\left(f_{1}\right)\right\|_{L^{p}\left(\omega, B\left(x_{0}, l\right)\right)} \leqslant C \omega\left(B\left(x_{0}, l\right)\right)^{\frac{1}{p}} \int_{2 l}^{\infty}\|f\|_{L^{p}\left(\omega, B\left(x_{0}, r\right)\right)} \omega\left(B\left(x_{0}, r\right)\right)^{-\frac{1}{p}} \frac{d r}{r} .
$$


When $1 \leqslant s^{\prime}<p<\infty$, set $v=p / s^{\prime}>1$. Since T satisfies (1.2), it follows from Hölder's inequality that

$$
\begin{aligned}
\sup _{x \in B\left(x_{0}, l\right)}\left|T\left(f_{2}\right)(x)\right| & \leqslant C \sum_{j=1}^{\infty}\left(\frac{1}{\left|B\left(x_{0}, 2^{j+1} l\right)\right|} \int_{B\left(x_{0}, 2^{j+1} l\right)}|f(y)|^{s^{\prime}} d y\right)^{\frac{1}{s^{\prime}}} \\
& \leqslant C \sum_{j=1}^{\infty}\left(2^{j+1} l\right)^{-\frac{n}{s^{\prime}}}\|f\|_{L^{p}\left(\omega, B\left(x_{0}, 2^{j+1} l\right)\right)}\left\|\omega^{-\frac{1}{p}}\right\|_{L^{s^{\prime} v^{\prime}}\left(B\left(x_{0}, 2^{j+1} l\right)\right)} \\
& \leqslant C \sum_{j=1}^{\infty} \int_{2^{j+1} l}^{2^{j+2} l}\left(2^{j+1} l\right)^{-\left(1+\frac{n}{s^{\prime}}\right)}\|f\|_{L^{p}\left(\omega, B\left(x_{0}, r\right)\right)}\left\|\omega^{-\frac{1}{\mathfrak{p}}}\right\|_{L^{s^{\prime} v^{\prime}}\left(B\left(x_{0}, r\right)\right)} d r \\
& \leqslant C \int_{2 l}^{\infty}\|f\|_{L^{p}\left(\omega, B\left(x_{0}, r\right)\right)}\left\|\omega^{-\frac{1}{p}}\right\|_{L^{s^{\prime} v^{\prime}}\left(B\left(x_{0}, r\right)\right)} \frac{d r}{r^{1+n / s^{\prime}}} .
\end{aligned}
$$

Note that $\omega \in A_{v}$, by (2.2) we get

$$
\left\|\omega^{-\frac{1}{\mathfrak{p}}}\right\|_{L^{s^{\prime} v^{\prime}}\left(B\left(x_{0}, r\right)\right)} \leqslant C r^{\frac{\mathfrak{n}}{s^{\prime}}} \omega\left(B\left(x_{0}, r\right)\right)^{-\frac{1}{\mathfrak{p}}} .
$$

Then

$$
\sup _{x \in B\left(x_{0}, l\right)}\left|T\left(f_{2}\right)(x)\right| \leqslant C \int_{2 l}^{\infty}\|f\|_{L^{p}\left(\omega, B\left(x_{0}, r\right)\right)} \omega\left(B\left(x_{0}, r\right)\right)^{-\frac{1}{p}} \frac{d r}{r} .
$$

When $s^{\prime}=p$, then $\omega \in A_{1}$. Then for any $p>1$,

$$
\begin{aligned}
\sup _{x \in B\left(x_{0}, l\right)}\left|T\left(f_{2}\right)(x)\right| & \leqslant C \sum_{j=1}^{\infty}\left(2^{j+1} l\right)^{-\frac{n}{p}}\left(\int_{B\left(x_{0}, 2^{j+1} l\right)}|f(y)|^{p} d y\right)^{\frac{1}{p}} \\
& \leqslant C \sum_{j=1}^{\infty}\left(2^{j+1} l\right)^{-\frac{n}{p}}\left(\int_{B\left(x_{0}, 2^{j+1} l\right)}|f(y)|^{p} w(x) d y\right)^{\frac{1}{p}}\left(\operatorname{essinf}_{x \in B\left(x_{0}, 2^{j+1} l\right)} w(x)\right)^{-\frac{1}{p}} \\
& \leqslant C \sum_{j=1}^{\infty} \int_{2^{j+1} l}^{j^{j+2} l}\|f\|_{L^{p}\left(\omega, B\left(x_{0}, 2^{j+1} l\right)\right)} \omega\left(B\left(x_{0}, 2^{j+1} l\right)\right)^{-\frac{1}{p}} \frac{d r}{r} \\
& \leqslant C \sum_{j=1}^{\infty} \int_{2^{j+1} l}^{j^{j+2} l}\|f\|_{L^{p}\left(\omega, B\left(x_{0}, r\right)\right)} \omega\left(B\left(x_{0}, r\right)\right)^{-\frac{1}{p}} \frac{d r}{r} \\
& \leqslant C \int_{2 l}^{\infty}\|f\|_{L^{p}\left(\omega, B\left(x_{0}, r\right)\right)} \omega\left(B\left(x_{0}, r\right)\right)^{-\frac{1}{p}} \frac{d r}{r} .
\end{aligned}
$$

By (2.5) and (2.6) we get

$$
\left\|T\left(f_{2}\right)\right\|_{L^{p}\left(\omega, B\left(x_{0}, l\right)\right)} \leqslant C \omega\left(B\left(x_{0}, l\right)\right)^{\frac{1}{p}} \int_{2 l}^{\infty}\|f\|_{L^{p}\left(\omega, B\left(x_{0}, r\right)\right.} \omega\left(B\left(x_{0}, r\right)\right)^{-\frac{1}{p}} \frac{d r}{r^{n+1}} .
$$

Combining (2.4) and (2.7), we complete the proof of Lemma 2.2.

Lemma 2.3. Suppose that $1 \leqslant s^{\prime} \leqslant p<\infty, p>1$, and $\omega \in A_{p / s^{\prime}}\left(\mathbb{R}^{n}\right)$. If $\mathrm{T}$ satisfies (1.2) and $[\mathrm{b}, \mathrm{T}]$ is bounded on $\mathrm{L}^{\mathrm{p}}\left(\omega, \mathbb{R}^{\mathfrak{n}}\right)$, then for any $l>0$, there is a constant $\mathrm{C}$ independent of $\mathrm{f}$ such that

$$
\mid[b, T](f)\left\|_{L^{p}\left(\omega, B\left(x_{0}, l\right)\right)} \leqslant C\right\| b\left\|_{*} \omega\left(B\left(x_{0}, l\right)\right)^{\frac{1}{p}} \int_{2 l}^{\infty}\right\| f \|_{L^{p}\left(\omega, B\left(x_{0}, r\right)\right)} \omega\left(B\left(x_{0}, r\right)\right)^{-\frac{1}{p}} \frac{d r}{r} .
$$

Proof. We represent $f$ as

$$
f(y)=f_{1}(y)+f_{2}(y), \quad f_{1}(y)=f(y) \chi_{B}\left(x_{0}, 2 l\right)(y) .
$$

Then

$$
\|[b, T](f)\|_{L^{p}\left(\omega, B\left(x_{0}, l\right)\right)} \leqslant\left\|[b, T]\left(f_{1}\right)\right\|_{L^{p}\left(\omega, B\left(x_{0}, l\right)\right)}+\left\|[b, T]\left(f_{2}\right)\right\|_{L^{p}\left(\omega, B\left(x_{0}, l\right)\right)} .
$$

Since $[b, T]$ is bounded on $L^{p}\left(\omega, \mathbb{R}^{\mathfrak{n}}\right)$, as the proof of (2.4) we get 


$$
\begin{aligned}
\left\|[b, T]\left(f_{1}\right)\right\|_{L^{p}\left(\omega, B\left(x_{0}, l\right)\right)} & \leqslant C\|b\|_{*}\|f\|_{L^{p}\left(\omega, B\left(x_{0}, 2 l\right)\right)} \\
& \leqslant C\|b\|_{*} \omega\left(B\left(x_{0}, l\right)\right)^{\frac{1}{p}} \int_{2 l}^{\infty}\|f\|_{L^{p}\left(\omega, B\left(x_{0}, r\right)\right)} \omega\left(B\left(x_{0}, r\right)\right)^{-\frac{1}{p}} \frac{d r}{r} .
\end{aligned}
$$

We now turn to deal with the term $\left\|[b, T]\left(f_{2}\right)\right\|_{L^{p}\left(\omega, B\left(x_{0}, l\right)\right)}$. For any given $x \in B\left(x_{0}, l\right)$, we have

$$
\begin{aligned}
\left|[b, T]\left(f_{2}\right)(x)\right| & \leqslant C\left|b(x)-b_{B\left(x_{0}, l\right)}\right|\left|T\left(f_{2}\right)(x)\right|+C\left|T\left(\left(b-b_{B\left(x_{0}, l\right)}\right) f_{2}\right)(x)\right| \\
& =I_{1}+I_{2} .
\end{aligned}
$$

Since T satisfies (1.2), by (2.5) and (2.6),

$$
I_{1} \leqslant C\left|b(y)-b_{B\left(x_{0}, l\right)}\right| \int_{2 l}^{\infty}\|f\|_{L^{p}\left(\omega, B\left(x_{0}, r\right)\right)} w\left(B\left(x_{0}, r\right)\right)^{-\frac{1}{p}} \frac{d r}{r} .
$$

Applying Lemma 2.1 we get

$$
\left\|I_{1} \mid\right\|_{L^{p}\left(\omega, B\left(x_{0}, l\right)\right)} \leqslant C\|b\|_{*} \omega\left(B\left(x_{0}, l\right)\right)^{\frac{1}{p}} \int_{2 l}^{\infty}\|f\|_{L^{p}\left(\omega, B\left(x_{0}, r\right)\right)} \omega\left(B\left(x_{0}, r\right)\right)^{-\frac{1}{p}} \frac{d r}{r} .
$$

On the other hand, it follows from (1.2) that

$$
I_{2} \leqslant C \sum_{j=1}^{\infty}\left(2^{j+1} l\right)^{-\frac{n}{s^{\prime}}}\left(\int_{B\left(x_{0}, 2^{j+1} l\right)}\left|\left(b(y)-b_{\left.B\left(x_{0}, l\right)\right)}\right) f(y)\right|^{s^{\prime}} d y\right)^{\frac{1}{s^{\prime}}} .
$$

Set $v=p / s^{\prime}$. From $\omega \in A_{v}$ we know $\omega^{1-v^{\prime}} \in A_{v^{\prime}}$. By Hölder's inequality

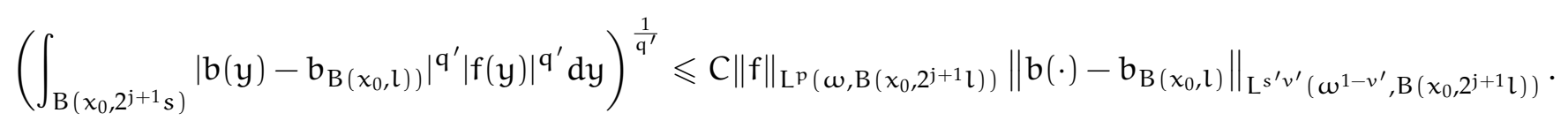

Consequently,

$$
\begin{aligned}
& I_{2} \leqslant \sum_{j=1}^{\infty} \int_{2^{j+1} l}^{2^{j+2} l}\left(2^{j+1} l\right)^{-\left(1+\frac{n}{s^{\prime}}\right)}\|f\|_{L^{p}\left(\omega, B\left(x_{0}, 2^{j+1} l\right)\right)}\left\|b(\cdot)-b_{B\left(x_{0}, l\right)}\right\|_{L^{s v^{\prime}}\left(\omega^{1-v^{\prime}}, B\left(x_{0}, 2^{j+1} l\right)\right)} d r \\
& \leqslant C \int_{2 l}^{\infty}\|f\|_{L^{p}\left(\omega, B\left(x_{0}, r\right)\right)}\left\|b(\cdot)-b_{B\left(x_{0}, l\right)}\right\|_{\left.L^{s^{\prime} v^{\prime}}\left(\omega^{1-v^{\prime}}, r\right)\right)} \frac{d r}{r^{1+n / s^{\prime}}} .
\end{aligned}
$$

Since $\omega^{-\frac{v^{\prime}}{s^{\prime}}}=\omega^{1-v^{\prime}} \in A_{v^{\prime}}$, we get

$$
\left(\omega^{1-v^{\prime}} B\left(x_{0}, r\right)\right)^{\frac{1}{s^{\prime} v^{\prime}}} \leqslant \operatorname{Cr}^{\frac{n}{v}}\left(\left(B\left(x_{0}, r\right)\right)^{-\frac{1}{p}} .\right.
$$

By Lemma 2.1 and the fact that $\omega \in A_{v}$, we obtain

$$
\begin{aligned}
\left(\int_{B\left(x_{0}, 2 j^{j+1} l\right)}\left|b(y)-b_{B\left(x_{0}, l\right)}\right|^{s^{\prime} v^{\prime}} \omega^{1-v^{\prime}}(y) d y\right)^{\frac{1}{s^{\prime} v^{\prime}}} & \leqslant C\|b\|_{*}\left(1+\ln \frac{r}{l}\right)\left(\omega^{1-v^{\prime}}\left(B\left(x_{0}, r\right)\right)\right)^{\frac{1}{s v^{\prime}}} \\
& \leqslant C\|b\|_{*} r^{\frac{n}{s^{\prime}}}\left(1+\ln \frac{r}{l}\right) \omega\left(B\left(x_{0}, r\right)\right)^{-\frac{1}{p}} .
\end{aligned}
$$

Then

$$
\mathrm{I}_{2} \leqslant \mathrm{C}\|\mathrm{b}\|_{*} \int_{2 l}^{\infty}\left(1+\ln \frac{\mathrm{r}}{\mathrm{l}}\right)\|\mathrm{f}\|_{\mathrm{L}^{\mathrm{p}}\left(\omega, \mathrm{B}\left(\mathrm{x}_{0}, \mathrm{r}\right)\right)} \omega\left(\mathrm{B}\left(\mathrm{x}_{0}, \mathrm{r}\right)\right)^{-\frac{1}{\mathrm{p}}} \frac{\mathrm{dr}}{\mathrm{r}}
$$

Therefore

$$
\left\|I_{2} \mid\right\|_{L^{p}\left(\omega, B\left(x_{0}, l\right)\right)} \leqslant C\|b\|_{*} \omega\left(B\left(x_{0}, l\right)\right)^{\frac{1}{p}} \int_{2 l}^{\infty}\left(1+\ln \frac{r}{l}\right)\|f\|_{L^{p}\left(\omega, B\left(x_{0}, r\right)\right)} \omega\left(B\left(x_{0}, r\right)\right)^{-\frac{1}{p}} \frac{d r}{r} .
$$




\section{Proof of Theorem 1.1}

Proof. For $f \in M_{\varphi_{1}}^{p}\left(w, \mathbb{R}^{n}\right)$, from the fact $\|f\|_{L^{p}\left(w, B\left(x_{0}, r\right)\right)}$ is a non-decreasing function of $r$, and

$$
(\underset{x \in E}{\operatorname{essinf}} f(x))^{-1}=\underset{x \in E}{\operatorname{essipsup}} \frac{1}{f(x)}
$$

holds for any real-valued nonnegative function $f$ and measurable on $E$ ([14, p.143]), we get

$$
\begin{aligned}
\frac{\|f\|_{L^{p}\left(\omega, B\left(x_{0}, r\right)\right)}}{\operatorname{essinf}_{0<r<t<\infty} \varphi_{1}\left(x_{0}, t\right) \omega\left(B\left(x_{0}, t\right)\right)^{\frac{1}{p}}} & \leqslant \operatorname{esssup}_{0<r<t<\infty} \frac{\|f\|_{L^{p}\left(\omega, B\left(x_{0}, r\right)\right)}}{\varphi_{1}\left(x_{0}, t\right) \omega\left(B\left(x_{0}, t\right)\right)^{\frac{1}{p}}} \\
& \leqslant \operatorname{esssup}_{t>0, x_{0} \in \mathbb{R}^{n}} \frac{\|f\|_{L^{p}\left(\omega, B\left(x_{0}, t\right)\right)}}{\varphi_{1}\left(x_{0}, t\right) \omega\left(B\left(x_{0}, t\right)\right)^{\frac{1}{p}}} \\
& \leqslant\|f\|_{M_{\varphi_{1}}^{p}\left(w, \mathbb{R}^{n}\right) .}
\end{aligned}
$$

Since $p>1$, and $\left(\varphi_{1}, \varphi_{2}\right)$ satisfies (1.3), we have

$$
\begin{aligned}
& \int_{l}^{\infty}\|f\|_{L^{p}\left(\omega, B\left(x_{0}, r\right)\right)} \omega\left(B\left(x_{0}, r\right)\right)^{-\frac{1}{p}} \frac{d r}{r} \\
& \quad \leqslant \int_{l}^{\infty} \frac{\|f\|_{L^{p}\left(\omega, B\left(x_{0}, r\right)\right)} \frac{\operatorname{essinf} \varphi_{1}\left(x_{0}, t\right) \omega\left(B\left(x_{0}, t\right)\right)^{\frac{1}{p}}}{\operatorname{essinf}_{r<t<\infty} \varphi_{1}\left(x_{0}, t\right) \omega\left(B\left(x_{0}, t\right)\right)^{\frac{1}{p}}} \frac{d r}{r}}{\omega\left(B\left(x_{0}, r\right)\right)^{\frac{1}{p}}} \frac{\left.d\left(x_{0}, t\right)\right)^{\frac{1}{p}}}{d r} \\
& \leqslant C\|f\|_{M_{\varphi_{1}}^{p}\left(w, \mathbb{R}^{n}\right)} \int_{l}^{\infty} \frac{\underset{r<t<\infty}{\operatorname{essinf}} \varphi_{1}\left(x_{0}, t\right) \omega\left(B\left(x_{0}, r\right)\right)^{\frac{1}{p}}}{\omega\left(B \left(x_{0}\right.\right.} \\
& \leqslant C\|f\|_{M_{\varphi_{1}}^{p}\left(w, \mathbb{R}^{n}\right)} \varphi_{2}\left(x_{0}, l\right) .
\end{aligned}
$$

Then by (2.3) we get

$$
\begin{aligned}
& \|\mathrm{T}(\mathrm{f})\|_{M_{\varphi_{2}}^{p}\left(w, \mathbb{R}^{n}\right)} \leqslant \mathrm{C} \sup _{x_{0} \in \mathbb{R}^{n}, l>0} \frac{1}{\varphi_{2}\left(x_{0}, l\right)}\left(\frac{1}{w\left(B\left(x_{0}, l\right)\right)} \int_{B\left(x_{0}, l\right)}|\mathrm{T}(f)(y)|^{p} \mathcal{w}(y) d y\right)^{1 / p} \\
& \leqslant C \sup _{x_{0} \in \mathbb{R}^{n}, l>0} \frac{1}{\varphi_{2}\left(x_{0}, l\right)} \int_{l}^{\infty}\|f\|_{L^{p}\left(\omega, B\left(x_{0}, r\right)\right)} \omega\left(B\left(x_{0}, r\right)\right)^{-\frac{1}{p}} \frac{d r}{r} \\
& \leqslant \mathrm{C}\|\mathrm{f}\|_{M_{\varphi_{1}}^{\mathrm{p}}\left(w, \mathbb{R}^{\mathrm{n}}\right)} \text {. }
\end{aligned}
$$

When $f \in M_{\varphi_{1}}^{p}\left(w, \mathbb{R}^{n}\right)$ and $\left(\varphi_{1}, \varphi_{2}\right)$ satisfies (1.4), then for $p>1$, we have

$$
\begin{aligned}
& \int_{l}^{\infty}\left(1+\ln \frac{r}{l}\right)\|f\|_{L^{p}\left(\omega, B\left(x_{0}, r\right)\right)} \omega\left(B\left(x_{0}, r\right)\right)^{-\frac{1}{p}} \frac{d r}{r}
\end{aligned}
$$

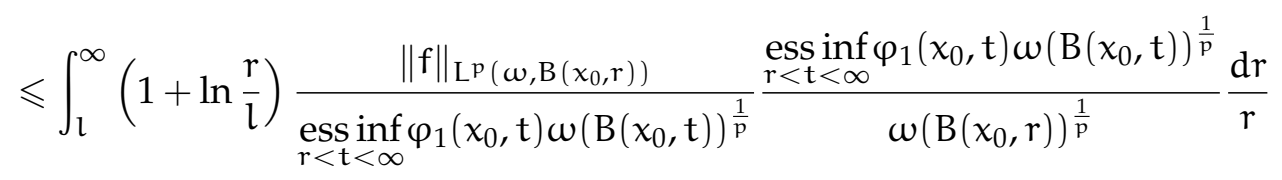

$$
\begin{aligned}
& \leqslant C\|f\|_{M_{\varphi_{1}}^{p_{1}}\left(w, \mathbb{R}^{n}\right)} \int_{l}^{\infty}\left(1+\ln \frac{r}{l}\right) \frac{\underset{r}{\mathrm{essinf}} \varphi_{1}\left(x_{0}, t\right) \omega\left(B\left(x_{0}, t\right)\right)^{\frac{1}{p}}}{\omega\left(B\left(x_{0}, r\right)\right)^{\frac{1}{p}}} \frac{d r}{r} \\
& \leqslant \mathrm{C}\|\mathbf{f}\|_{M_{\varphi_{1}}^{\mathrm{p}}\left(w, \mathbb{R}^{\mathfrak{n}}\right)} \varphi_{2}\left(\mathrm{x}_{0}, \mathrm{l}\right) \text {. }
\end{aligned}
$$


By (2.8) we get

$$
\begin{aligned}
& \|[b, T](f)\|_{M_{\varphi_{2}}^{p_{2}\left(w, \mathbb{R}^{n}\right)} \leqslant C} \sup _{x_{0} \in \mathbb{R}^{n}, l>0} \frac{1}{\varphi_{2}\left(x_{0}, l\right)}\left(\frac{1}{w\left(B\left(x_{0}, l\right)\right)} \int_{B\left(x_{0}, l\right)}\left|\left[b, T_{\Omega}\right](f)(y)\right|^{p} \mathcal{w}(y) d y\right)^{1 / p} \\
& \leqslant C \sup _{x_{0} \in \mathbb{R}^{n}, l>0} \frac{1}{\varphi_{2}\left(x_{0}, l\right)} \int_{s}^{\infty}\left(1+\ln \frac{r}{l}\right)\|f\|_{L^{p}\left(\omega, B\left(x_{0}, r\right)\right)} \omega\left(B\left(x_{0}, r\right)\right)^{-\frac{1}{p}} \frac{d r}{r} \\
& \leqslant \mathrm{C}\|\mathbf{f}\|_{M_{\varphi_{1}}^{\mathrm{p}}\left(w, \mathbb{R}^{\mathfrak{n}}\right)} \text {. }
\end{aligned}
$$

\section{Some applications}

In this section, we shall apply Theorem 1.1 to several particular operators such as singular integral operators with rough kernel, Bochner-Riesz operators and Marcinkiewicz integral operators.

\subsection{Singular integral operators with rough kernels}

Suppose that $\mathbb{S}^{n-1}$ is the unit sphere in $\mathbb{R}^{n}(n \geqslant 2)$ equipped with the normalized Lebesgue measure do. Let $\Omega \in \mathrm{L}^{\mathrm{s}}\left(\mathrm{S}^{n-1}\right)$ with $1<\mathrm{s}<\infty$ be homogeneous of degree zero and satisfy the cancellation condition

$$
\int_{S^{n-1}} \Omega\left(x^{\prime}\right) d \sigma\left(x^{\prime}\right)=0,
$$

where $x^{\prime}=x /|x|$ for any $x \neq 0$. The homogeneous singular integral operator $T_{\Omega}$ is defined by

$$
T_{\Omega} f(x)=\lim _{\epsilon \rightarrow 0} \int_{|y|>\epsilon} \frac{\Omega\left(y^{\prime}\right)}{|y|^{n}} f(x-y) d y .
$$

Let $b$ be a locally integrable function on $\mathbb{R}^{n}$, the commutator of $b$ and $T_{\Omega}$ is defined by

$$
\left[b, T_{\Omega}\right] f(x)=b(x) T_{\Omega} f(x)-T_{\Omega}(b f)(x) .
$$

Following [5], there is a constant $C$ independent of $f$ such that

$$
\left\|T_{\Omega} f\right\|_{L^{p}\left(\omega, \mathbb{R}^{n}\right)} \leqslant C\|f\|_{L^{p}\left(\omega, \mathbb{R}^{n}\right)}
$$

for every $s^{\prime} \leqslant p<\infty$ and $\omega \in A_{p / s^{\prime}}$. By the well-known boundedness criterion for the commutators of linear operators, which was obtained by Alvarez et al. (see [1]), we see that

$$
\left\|\left[b, T_{\Omega}\right] f\right\|_{L^{p}\left(\omega, \mathbb{R}^{n}\right)} \leqslant C\|b\|_{*}\|f\|_{L^{p}\left(\omega, \mathbb{R}^{n}\right)},
$$

holds for all $b \in B M O, s^{\prime} \leqslant p<\infty$ and $w \in A_{p} / s^{\prime}$.

Theorem 4.1. Suppose $\Omega \in \mathrm{L}^{\mathrm{s}}\left(\mathrm{S}^{n-1}\right)$ with $1<\mathrm{s}<\infty$. Let $\mathrm{s}^{\prime} \leqslant \mathrm{p}<\infty, \omega \in A_{\mathrm{p} / \mathrm{s}^{\prime}}$ and $\mathrm{b} \in \mathrm{BMO}\left(\mathbb{R}^{\mathrm{n}}\right)$. If $\left(\varphi_{1}, \varphi_{2}\right)$ satisfies the condition (1.3), then there is a constant $\mathrm{C}>0$ independent of $\mathrm{f}$ such that

$$
\left\|T_{\Omega} f\right\|_{M_{\varphi_{2}}^{p}\left(\omega, \mathbb{R}^{n}\right)} \leqslant C\|f\|_{M_{\varphi_{1}}^{p}\left(\omega, \mathbb{R}^{n}\right)} .
$$

If $\left(\varphi_{1}, \varphi_{2}\right)$ satisfies the condition (1.4), then there is a constant $\mathrm{C}>0$ independent of $\mathrm{f}$ such that

$$
\left\|\left[b, T_{\Omega}\right] f\right\|_{M_{\varphi_{2}}^{p}\left(w, \mathbb{R}^{n}\right)} \leqslant C\|b\|_{*}\|f\|_{M_{\varphi_{1}}^{p}\left(w, \mathbb{R}^{n}\right)} .
$$


Proof. We only need to prove $\mathrm{T}_{\Omega}$ satisfies (1.2). By Hölder's inequality,

$$
\begin{aligned}
\sup _{x \in B\left(x_{0}, l\right)}\left|T_{\Omega}\left(f \chi_{\left(B\left(x_{0}, 2 l\right)^{c}\right.}\right)(x)\right| \leqslant & \sup _{x \in B\left(x_{0}, l\right)}\left|\int_{B\left(x_{0}, 2 l\right)^{c}} \frac{\Omega\left((x-y)^{\prime}\right)}{|x-y|^{n}} f(y) d y\right| \\
\leqslant & \sup _{x \in B\left(x_{0}, l\right)} \sum_{j=1}^{\infty}\left(\int_{B\left(x_{0}, 2^{j+1} l\right) \backslash B\left(x_{0}, 2^{j} l\right)}\left|\Omega\left((x-y)^{\prime}\right)\right|^{s} d y\right)^{\frac{1}{s}} \\
& \times\left(\int_{B\left(x_{0}, 2^{j+1} l\right) \backslash B\left(x_{0}, 2^{j} l\right)} \frac{|f(y)|^{s^{\prime}}}{|x-y|^{n s^{\prime}}} d y\right)^{\frac{1}{s^{\prime}}} .
\end{aligned}
$$

When $x \in B\left(x_{0}, l\right)$ and $y \in B\left(x_{0}, 2^{j+1} l\right) \backslash B\left(x_{0}, 2^{j} l\right)$, by a direct calculation, we can see that $2^{j-1} l \leqslant|y-x|<$ $2^{j+1} l$. Hence

$$
\left(\int_{B\left(x_{0}, 2^{j+1} l\right) \backslash B\left(x_{0}, 2^{j} l\right)}\left|\Omega\left((x-y)^{\prime}\right)\right|^{s} d y\right)^{\frac{1}{s}} \leqslant C\|\Omega\|_{L^{s}\left(S^{n-1}\right)}\left|B\left(x_{0}, 2^{j+1} l\right)\right|^{\frac{1}{s}} .
$$

We also note that if $x \in B\left(x_{0}, l\right), y \in B\left(x_{0}, 2 l\right)^{c}$, then $|y-x| \sim\left|y-x_{0}\right|$. Consequently

$$
\left(\int_{B\left(x_{0}, 2^{j+1} l\right) \backslash B\left(x_{0}, 2^{j} l\right)} \frac{|f(y)|^{s^{\prime}}}{|x-y|^{n s^{\prime}}} d y\right)^{\frac{1}{s^{\prime}}} \leqslant \frac{1}{\left|B\left(x_{0}, 2^{j+1} l\right)\right|}\left(\int_{B\left(x_{0}, 2^{j+1} l\right)}|f(y)|^{s^{\prime}} d y\right)^{\frac{1}{s^{\prime}}} .
$$

Combining (4.1) and (4.2), we get

$$
\sup _{x \in B\left(x_{0}, l\right)}\left|T_{\Omega}\left(f \chi_{\left(B\left(x_{0}, 2 l\right)^{c}\right.}\right)(x)\right| \leqslant C \sum_{j=1}^{\infty}\left(2^{j+1} l\right)^{-\frac{n}{s^{\prime}}}\left(\int_{B\left(x_{0}, 2^{j+1} l\right)}|f(y)|^{s^{\prime}} d y\right)^{\frac{1}{s^{\prime}}} .
$$

\subsection{Bochner-Riesz operators}

Bochner-Riesz operators were first introduced by Bochner [2] in connection with summation of multiple Fourier series and played an important role in harmonic analysis. The Bochner-Riesz operators of order $\delta>0$ in $\mathbb{R}^{n}(n \geqslant 2)$ are defined initially for Schwartz functions in terms of Fourier transforms by

$$
\left(T_{R}^{\delta} f\right)^{\wedge}(\xi)=\left(1-\frac{|\xi|^{2}}{R^{2}}\right)_{+}^{\delta} \hat{f}(\xi),
$$

where $\hat{f}$ denotes the Fourier transform of $f$. These operators can be expressed as convolution operators by the formula

$$
T_{R}^{\delta} f(x)=\left(f * \phi_{1 / R}\right)(x),
$$

where $\phi_{1 / R}(x)=R^{n} f(R x)$, and for all $\delta \geqslant(n-1) / 2$,

$$
|\phi(x)| \leqslant \frac{C}{(1+|x|)^{\frac{n+1}{2}+\delta}} .
$$

The associated maximal Bochner-Riesz operator is defined by

$$
T_{*}^{\delta}(f)(x)=\sup _{R>0}\left|T_{R}^{\delta} f(x)\right| .
$$

When $\delta>(n-1) / 2$, it is well-known that ([13])

$$
\mathrm{T}_{*}^{\delta}(f)(x) \leqslant \operatorname{CM}(f)(x) .
$$


By [12], if $\delta=(n-1) / 2$ and $\omega \in A_{p}$, then there exists a constant $C>0$ such that

$$
\left\|T_{*}^{\delta}(f)\right\|_{L^{p}(\omega)} \leqslant C\|f\|_{L^{p}(\omega)}, \quad \text { for } 1<p<\infty .
$$

Then, by the boundedness of maximal function $M(f)$ on $L^{p}(\omega)$, we know that if $\omega \in A_{p}(1<p<\infty)$, then for all $\delta \geqslant(n-1) / 2$,

$$
\left\|T_{*}^{\delta}(f)\right\|_{L^{p}(\omega)} \leqslant C\|f\|_{L^{p}(\omega)}
$$

holds.

Let $b$ be a locally integrable function on $\mathbb{R}^{n}$, for any given $R>0$, the commutator of $b$ and $T_{R}^{\delta}$ is defined as follows

$$
\left[b, T_{R}^{\delta}\right] f(x)=b(x) T_{R}^{\delta} f(x)-T_{R}^{\delta}(T f)(x) .
$$

Note that $T_{R}^{\delta} f(x) \leqslant T_{*}^{\delta}(f)(x)$, then, if $\omega \in A_{p}(1<p<\infty)$, the equality

$$
\left\|T_{R}^{\delta}(f)\right\|_{L^{p}(\omega)} \leqslant C\|f\|_{L^{p}(\omega)}
$$

holds for all $\delta \geqslant(n-1) / 2$. Therefore, by the boundedness criterion for the commutators of linear operators, we see that if $b \in B M O$, then $\left[b, T_{R}^{\delta}\right]$ is also bounded on $L^{p}(\omega)$ for all $1<p<\infty$ and $\omega \in A_{p}$.

Theorem 4.2. Suppose $\delta \geqslant(n-1) / 2$ and $1<p<\infty$. Let $b \in B M O$, and let $\omega \in A_{p}$. If $\left(\varphi_{1}, \varphi_{2}\right)$ satisfies the condition (1.3), then there is a constant $\mathrm{C}>0$ independent of $\mathrm{f}$ such that

$$
\left\|T_{*}^{\delta} f\right\|_{M_{\varphi_{2}}^{p}\left(\omega, \mathbb{R}^{n}\right)} \leqslant C\|f\|_{M_{\varphi_{1}}^{p}\left(\omega, \mathbb{R}^{n}\right)} .
$$

If $\left(\varphi_{1}, \varphi_{2}\right)$ satisfies the condition (1.4), then there is a constant $C>0$ independent of $\mathrm{f}$ such that

$$
\left\|\left[b, T_{R}^{\delta}\right] f\right\|_{M_{\varphi_{2}}^{p}\left(\omega, \mathbb{R}^{n}\right)} \leqslant C\|b\|_{*}\|f\|_{M_{\varphi_{1}}^{p}\left(\omega, \mathbb{R}^{n}\right)} .
$$

Proof. Note that when $\delta \geqslant(n-1) / 2$, then by the estimate (4.3), we have

$$
|\phi(x)| \leqslant \frac{C}{|x|^{n}} .
$$

We also observe that when $x \in B\left(x_{0}, l\right), y \in\left(B\left(x_{0}, 2 l\right)\right)^{c}$, then $|x-y| \sim\left|x-x_{0}\right|$. Hence

$$
\begin{aligned}
\sup _{x \in B\left(x_{0}, l\right)}\left|T_{R}^{\delta}\left(f \chi_{\left(B\left(x_{0}, 2 l\right)\right)^{c}}\right)(x)\right| & \leqslant \sup _{x \in B\left(x_{0}, l\right)} T_{*}^{\delta}\left(f \chi_{\left(B\left(x_{0}, 2 l\right)\right)^{c}}\right)(x) \\
& =C \sup _{x \in B\left(x_{0}, l\right)} \sup _{R>0} \mid\left(f \chi_{\left.\left(B\left(x_{0}, 2 l\right)\right)^{c}\right) * \phi_{1 / R}(x) \mid}\right. \\
& \leqslant C \sup _{x \in B\left(x_{0}, l\right) R>0} \sup _{\left(B\left(x_{0}, 2 l\right)\right)^{c}} \frac{R^{n}}{(R|x-y|)^{n}}|f(y)| d y \\
& \leqslant C \sum_{j=1}^{\infty} \frac{1}{\left|B\left(x_{0}, 2^{j+1} l\right)\right|} \int_{B\left(x_{0}, 2^{j+1} l\right)}|f(y)| d y .
\end{aligned}
$$

This means that $\mathrm{T}_{\mathrm{R}}^{\delta}$ and $\mathrm{T}_{*}^{\delta}$ satisfy (1.2).

\subsection{Marcinkiewicz integral operators}

Suppose that $\mathrm{S}^{n-1}$ is the unit sphere in $\mathbb{R}^{n}(n \geqslant 2)$ equipped with the normalized Lebesgue measure do. Let $\Omega \in \mathrm{L}^{\mathrm{s}}\left(\mathrm{S}^{n-1}\right)$ with $1<s \leqslant \infty$ be homogeneous of degree zero and satisfy the cancellation condition

$$
\int_{S^{n-1}} \Omega\left(x^{\prime}\right) d \sigma\left(x^{\prime}\right)=0,
$$

where $x^{\prime}=x /|x|$ for any $x \neq 0$. The Marcinkiewicz integral of higher dimension $\mu_{\Omega}$ is defined by 


$$
\mu_{\Omega}(f)(x)=\left(\int_{0}^{\infty}\left|F_{\Omega, t}(x)\right|^{2} \frac{d t}{t^{3}}\right)^{1 / 2}
$$

where

$$
F_{\Omega, t}(x)=\int_{|x-y| \leqslant t} \frac{\Omega(x-y)}{|x-y|^{n-1}} f(y) d y .
$$

We will also consider the commutator generated by Marcinkiewcz integral $\mu_{\Omega}$ and $b$ is defined as follows

$$
\left[b, \mu_{\Omega}\right](f)(x)=\left(\int_{0}^{\infty}\left|\int_{|x-y| \leqslant t} \frac{\Omega(x-y)}{|x-y|^{n-1}}(b(x)-b(y)) f(y) d y\right|^{2} \frac{d t}{t^{3}}\right)^{\frac{1}{2}} .
$$

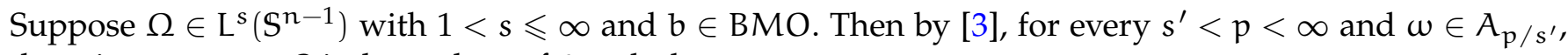
there is a constant $C$ independent of $f$ such that

$$
\left\|\mu_{\Omega}(f)\right\|_{L^{p}\left(\omega, \mathbb{R}^{\mathfrak{n}}\right)} \leqslant C\|f\|_{L^{p}\left(\omega, \mathbb{R}^{\mathfrak{n}}\right)} .
$$

By [4], for every $s^{\prime}<p<\infty$ and $\omega \in A_{p / s^{\prime}}$, there is a constant $C$ independent of $f$ such that

$$
\left\|\left[b, \mu_{\Omega}\right](f)\right\|_{L^{p}\left(\omega, \mathbb{R}^{n}\right)} \leqslant C\|b\|_{*}\|f\|_{L^{p}\left(\omega, \mathbb{R}^{n}\right)} .
$$

Theorem 4.3. Suppose that $\Omega \in \mathrm{L}^{\mathrm{s}}\left(\mathrm{S}^{n-1}\right)$ with $1<\mathrm{s} \leqslant \infty$. Let $\mathrm{s}^{\prime}<\mathrm{p}<\infty, \omega \in \mathrm{A}_{\mathrm{p} / \mathrm{s}^{\prime}}$ and $\mathrm{b} \in \mathrm{BMO}$. If $\left(\varphi_{1}, \varphi_{2}\right)$ satisfies the condition (1.3), then there is a constant $\mathrm{C}>0$ independent of $\mathrm{f}$ such that

$$
\left\|\mu_{\Omega} f\right\|_{M_{\varphi_{2}}^{p}\left(\omega, \mathbb{R}^{n}\right)} \leqslant C\|f\|_{M_{\varphi_{1}}^{p}\left(\omega, \mathbb{R}^{n}\right)} .
$$

If $\left(\varphi_{1}, \varphi_{2}\right)$ satisfies the condition (1.4), then there is a constant $C>0$ independent of $\mathrm{f}$ such that

$$
\left\|\left[b, \mu_{\Omega}\right] f\right\|_{M_{\varphi_{2}}^{p}\left(\omega, \mathbb{R}^{n}\right)} \leqslant C\|b\|_{*}\|f\|_{M_{\varphi_{1}}^{p}\left(\omega, \mathbb{R}^{n}\right)} .
$$

Proof. Observe that when $x \in B\left(x_{0}, l\right)$ and $y \in B\left(x_{0}, 2^{j+1} l\right) \backslash B\left(x_{0}, 2^{j} l\right)(j \geqslant 1)$, then

$$
t \geqslant|x-y| \geqslant\left|y-x_{0}\right|-\left|x-x_{0}\right| \geqslant 2^{j-1} l .
$$

Then, by Minkowski's inequality we have

$$
\begin{aligned}
& \mu_{\Omega}\left(f \chi_{\left(B\left(x_{0}, 2 l\right)\right)^{c}}\right)(x)=\left(\int_{0}^{\infty}\left|\int_{B\left(x_{0}, 2 l\right)^{c} \cap\{y:|x-y| \leqslant t\}} \frac{\Omega(x-y)}{|x-y|^{n-1}} f(y) d y\right|^{2} \frac{d t}{t^{3}}\right)^{\frac{1}{2}} \\
& =\left(\int_{0}^{\infty}\left|\sum_{j=1}^{\infty} \int_{\left(B\left(x_{0}, 2^{j+1} l\right) \backslash B\left(x_{0}, 2^{j} l\right)\right)^{\mathrm{c}} \cap\{y:|x-y| \leqslant t\}} \frac{\Omega(x-y)}{|x-y|^{\mathrm{n}-1}} f(y) d y\right|^{2} \frac{d t}{t^{3}}\right)^{\frac{1}{2}} \\
& \leqslant C \sum_{j=1}^{\infty}\left(\int_{B\left(x_{0}, 2^{j+1} l\right) \backslash B\left(x_{0}, 2^{j} l\right)} \frac{|\Omega(x-y)|}{|x-y|^{n-1}}|f(y)| d y\right)\left(\int_{2^{j-1} l}^{\infty} \frac{d t}{t^{3}}\right)^{\frac{1}{2}} \\
& \leqslant C \sum_{j=1}^{\infty}\left(2^{j+1} l\right)^{-1} \int_{B\left(x_{0}, 2^{j+1} l\right) \backslash B\left(x_{0}, 2^{j} l\right)} \frac{|\Omega(x-y)|}{|x-y|^{n-1}}|f(y)| d y .
\end{aligned}
$$

When $\Omega \in \mathrm{L}^{\infty}\left(\mathrm{S}^{\mathrm{n}-1}\right)$, then

$$
\sup _{x \in B\left(x_{0}, l\right)} \mu_{\Omega}\left(f \chi_{\left(B\left(x_{0}, 2 l\right)\right)^{c}}\right)(x) \leqslant C \sum_{j=1}^{\infty}\left(2^{j+1} l\right)^{-n} \int_{B\left(x_{0}, 2^{j+1} l\right)}|f(y)| d y .
$$


When $\Omega \in \mathrm{L}^{\mathrm{s}}\left(\mathrm{S}^{\mathrm{n}-1}\right), 1<\mathrm{s}<\infty$, then by Hölder's inequality,

$$
\begin{aligned}
& \int_{B\left(x_{0}, 2^{j+1} l\right) \backslash B\left(x_{0}, 2^{j} l\right)} \frac{|\Omega(x-y)|}{|x-y|^{n-1}}|f(y)| d y \\
& \quad \leqslant C\left(\int_{B\left(x_{0}, 2^{j+1} l\right) \backslash B\left(x_{0}, 2^{j} l\right)}\left|\Omega\left((x-y)^{\prime}\right)\right|^{s} d y\right)^{1 / s}\left(\int_{B\left(x_{0}, 2^{j+1} l\right) \backslash B\left(x_{0}, 2^{j} l\right)} \frac{|f(y)|^{s^{\prime}}}{|x-y|^{(n-1) s^{\prime}}} d y\right)^{1 / s^{\prime}} .
\end{aligned}
$$

It follows from (4.1), (4.4) and (4.6) that

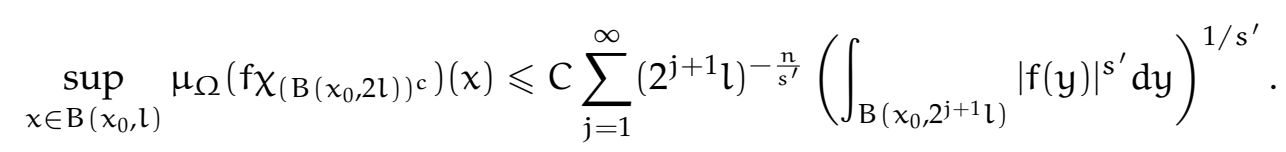

Combining (4.5) with (4.7), the proof of Theorem 4.3 is completed.

\section{References}

[1] J. Álvarez, R. J. Bagby, D. S. Kurtz, C. Pérez, Weighted estimates for commutators of linear operators, Studia Math., 104 (1993), 195-209. 4.1

[2] S. Bochner, Summation of multiple Fourier series by spherical means, Trans. Amer. Math. Soc, 40 (1936), 175-207. 4.2

[3] Y. Ding, D.-S. Fan, Y.-B. Pan, Weighted boundedness for a class of rough Marcinkiewicz integrals, Indiana Univ. Math. J., 48 (1999), 1037-1055. 4.3

[4] Y. Ding, S.-Z. Lu, K. Yabuta, On commutators of Marcinkiewicz integrals with rough kernel, J. Math. Anal. Appl, 275 (2002), 60-68. 4.3

[5] J. Duoandikoetxea, Weighted norm inequalities for homogeneous singular integrals, Trans. Amer. Math. Soc., 336 (1993), 869-880. 4.1

[6] Y. Hu, Y.-S. Wang, Multilinear fractional integral operators on generalized weighted Morrey spaces, J. Inequal. Appl., 2014 (2014), 18 pages. 2.1

[7] F. John, L. Nirenberg, On functions of bounded mean oscillation, Comm. Pure Appl. Math., 14 (1961), 415-426. 2

[8] Y. Komori, S. Shirai, Weighted Morrey spaces and a singular integral operator, Math. Nachr., 289 (2009), 219-231. 1

[9] T. Mizuhara, Boundedness of some classical operators on generalized Morrey spaces, Harmonic analysis, Sendai, (1990), ICM-90 Satell. Conf. Proc., Springer, Tokyo, (1991), 183-189. 1

[10] C. B. Morrey, Jr., On the solutions of quasi-linear elliptic partial differential equations, Trans. Amer. Math. Soc., 43 (1938), 126-166. 1

[11] B. Muckenhoupt, Weighted norm inequalities for the Hardy maximal function, Trans. Amer. Math. Soc., 165 (1972), 207-226. 2

[12] X. L. Shi, Q. Y. Sun, Weighted norm inequalities for Bochner-Riesz operators and singular integral operators, Proc. Amer. Math. Soc., 116 (1992), 665-673. 4.2

[13] E. M. Stein, G. Weiss, Introduction to Fourier analysis on Euclidean spaces, Princeton Mathematical Series, Princeton University Press, Princeton, N.J., (1971). 4.2

[14] R. L. Wheeden, A. Zygmund, Measure and integral, An introduction to real analysis. Pure and Applied Mathematics, Marcel Dekker, Inc., New York-Basel, (1977). 3 